\title{
Study on the Features and Strategies of Number-Concept Development of Rural Children in Early Primary School Years: Study of Number-Concept Development of Rural Children
}

\author{
$\mathrm{He} \mathrm{Li}^{1,2}$, Mengxu Fan ${ }^{3,4}$, Christopher Hermann ${ }^{5}$ \\ ${ }^{1}$ School of Education Science, Shanxi University, Shanxi, PR China \\ ${ }^{2}$ School of Education, Minzu University of China, Beijing, PR China \\ ${ }^{3}$ School of Foreign Studies, Minzu University of China, Beijing, PR China \\ ${ }^{4}$ University of Melbourne, Melbourne, Australia \\ ${ }^{5}$ University of New England, Armidale, Australia \\ Correspondence: He Li, School of Education Science, Shanxi University, Taiyuan, Shanxi, PR China.
}

Received: June 12, $2020 \quad$ Accepted: July 19, $2020 \quad$ Online Published: July 20, 2020

doi:10.11114/ijce.v3i2.4894

URL: https://doi.org/10.11114/ijce.v3i2.4894

\begin{abstract}
The development level of children's number-concept is a critical element in measuring the development of children's thinking. Previous studies have shown that preschool is an important stage of development and that vocabulary can help promote children's number-concept development. This study aims to explore the characteristics of the development of rural children's number-concept in their early years, and propose teaching strategies suitable for rural children based on the research results. This research method is based on a one-to-one standard test, supplemented by a self-designed structured interview for teachers. The research object is the early school-age children in rural areas. The impact of gender, age, school, and language on children's number-concept development is discussed. By describing and studying the characteristics of their number-concept development, educational strategies and suggestions are put forward. This research can contribute to the literature theoretically, pedagogically and practically.
\end{abstract}

Keywords: number-concept, early primary school years, rural areas, teaching strategies

\section{Introduction}

Number-concept, or number sense is defined as a "good intuition about numbers and their relationships. It develops gradually as a result of exploring numbers, visualizing them in a variety of contexts, and relating them in ways that are not limited by traditional algorithms" (Howden, 1989). Baroody and Ginsburg suggested categorizing children's mathematical skills into two types: informal and formal. While the formal skills mainly refer to mathematical knowledge taught in school, the informal skills include numerical concepts acquired before children enter school, such as counting words, enumeration, cardinality rules, and quantitative comparison (Baroody \& Ginsburg, 1982). Ansari et al. believed that children's understanding of the cardinality principle is driven more by visuo-spatial competence rather than their language ability (Ansari et al., 2003). Overall, however, the research on children's mathematical education in early school years is largely lagging behind that on children's literacy education, although recent years have seen an increasing number of studies on pedagogical interventions in children's mathematical development (Greenes, Ginsburg, \& Balfanz., 2004; Griffin, Case, \& Siegler, 1994; Starkey \& Klein, 2000). In the development of intervention studies, the connection between mathematical and vocabulary abilities is likely to be a key issue in young children's mathematical education. In 2012, James Negen and Barbara W. Sarnecka's research found that there is a significant correlation between children's number-concept acquisition and general vocabulary of their first language (L1), no matter how old the children are. The finding showed that having a larger nominal vocabulary in fact helps children to learn number words, which is contrary to the opinion expressed by Ansari et al. (2003). They also argued that compared with early literacy, attention skills, socioemotional skills, family background measures, or IQ, early math knowledge is a better predictor of children's achievement later in life. Another earlier well-known meta-analysis consisting of six longitudinal datasets including more than 34,000 students even maintained that pre-kindergarten mathematical skills are the single best predictor of later achievement (Duncan et al., 2007). From the studies mentioned above, it may be 
implied that interventions aiming to build young children's vocabulary can be a potential help for developing number concept. Therefore, number-concept acquisition of preschoolers should be a crucial topic for educators and researchers.

Based on previous studies, this paper chooses children of rural areas in their first year of primary school as participants, for two main reasons: first, previous studies find that the gap of number-concept development between cities and rural areas is very evident, therefore requiring more intervention for rural students; second, in rural areas, Mandarin is not as popular as the local dialect where the children live, which results in a bilingual environment (for example, dialect in communicating with family and friends, mandarin in television, school teaching, internet, etc.). With these considerations in mind, this research includes first-year primary school children living in multiethnic rural areas as participants. On the one hand, they are influenced by a multilingual environment, which is supposed to be an advantage for number-concept development; on the other hand, most of them lack proper pre-school education, which might be a disadvantage (Zhao \& Jiang, 2011; Xie, Tian, \& Lv, 2014). Due to these characteristics, these participants are representative in Chinese rural areas. This study, focusing on the research and description of the features in number-concept development of Chinese rural early-year children, will enrich the theoretical exploration of young children's education, and will also help to find a way to improve rural children's mathematical skills, even alleviating poverty with education in the long term.

\section{Method}

\subsection{Participants}

A sample of 220 children in their first year of primary school were recruited from three rural schools in S town, K city, Y province (Note 1) (which is situated at the Chinese southwest border). All of these children came from rural families near the three schools. The 220 children were tested individually by the tester, and 214 valid results were collected after evaluation, including 34 from village school B, 138 from town school C, and 42 from town school D. Among them, there are 90 male and 124 female students. In terms of age, 80 children were at age 6,116 at 7, and 18 at the age of 8 , which put the overall sample average age at 6.71. In addition, 12 valid interviews with the school teachers were collected during the study.

\subsection{Measures}

The standard test used in this study was the Student Number-concept Assessment Test, designed on the basis of the Test of Early Mathematics Ability (Ginsburg \& Baroody, 1990), the Number Sense Test (Malofeeva, Day, Saco, Young, \& Ciancio, 2004), and the Test of Children's Numericity Estimation and Number Sense (Zhao \& Zhou, 2006), and supplemented by unstructured interviews of teachers. The test aims to examine children's mathematical abilities by verbal protocol. In or after the process of being tested, participants were asked to report orally how they think and calculate when solving mathematical questions. By recording and analyzing their statements and the strategies they used, participants' psychological activities and thinking patterns were revealed. The Number-concept Assessment Test conducted was comprised of six parts: number counting skills, cardinality concept, ordinal number concept, quantitative comparison, quantity conservation concept, and number operation.

\subsection{Reliability Analysis and Validity Analysis}

Since this study intended to discover the correlations between different items based on the same test, Cronbach's alpha in internal consistency was the applicable indicator. According to the Reliability Analysis in SPSS 22.0, $\alpha=0.862$, which meant this test is highly reliable. By varimax rotation, the principal factor analysis of the 30 questions in the Number-concept Assessment Test showed that $\mathrm{KMO}=0.761$, and the Bartlett test for sphericity was statistically significant $(\mathrm{p}<0.001)$. The reliability analysis indicated that the test result was legitimate for factor analysis, with a total variance of $74.53 \%$, which meant the test had high validity.

\section{Results Analysis}

\subsection{Descriptive Statistics of the Number-concept Assessment Test for Rural Children in Their Early Primary School Years}

The full marks for the Number-concept Assessment Test were 76 points, which contained 6 sections, namely number counting skills, cardinality concept, ordinal number concept, quantitative comparison, quantity conservation concept, and number operation as mentioned earlier. The average score of the participants was 30.40 , with the highest 73 , the lowest 3 and the standard deviation 12.96. As is shown in Figure 1, the frequency distribution showed negative skewness, indicating that the overall score was low and the differences between individuals were large. 


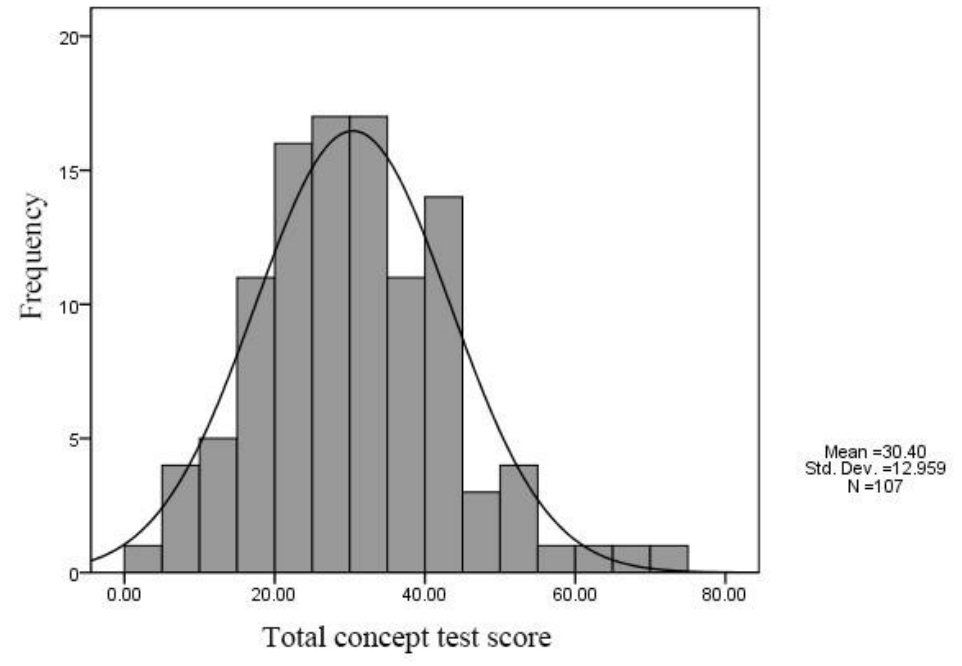

Figure 1. Distribution of the total number of rural children in early school age groups

\subsubsection{Counting Skills}

The full marks of this section, which includes counting on and back, naming the neighboring number, and skip counting, were 25 points. In the test, the average score was 7.61 and the standard deviation 5.76. In "counting on" related questions, $42.1 \%$ of the students could not count to 100 , and only $5.2 \%$ of them could count to more than 110 , which showed that young children in the first year of their primary schooling could not draw inferences about other cases from one instance before they were taught by teachers. In "naming the neighboring number" questions, $77.0 \%$ of the children who did not get the correct number answered wrongly because of mixing up "the previous number" and "the next number". This proved that they were not very clear on the notion of "neighboring numbers", especially the notion of "previous" and "next". A preliminary analysis of the causes showed that it was related to the lingual and cultural environment of where the rural children lived. The test results about "counting back" were polarized. Some children fully mastered the skill, while the others could not do it at all. But overall, most participants did not perform well in this part. In addition, it was found that $72.9 \%$ of the children could not understand what "skip counting" is, while only $3.7 \%$ of them could skip count to higher than 50 .

\subsubsection{Cardinality Concept}

The 4 questions in this section had a full mark of 8 points with the average score being 4.95 and the standard deviation 2.38. In question 5 (the first one in this section), participants were asked to count using 50 buttons as assistance. The result showed that $71.0 \%$ of them could count correctly. In question 6 , where the experimenter did an exemplary counting from a number other than 1, and asked the participants if that was correct, there were $40.2 \%$ of participants answering correctly. Question 7 asked the students to first observe the examiner counting a straight line of buttons starting from the middle or a few random distributed buttons, and then asked participants to predict whether the counting results would be the same. $76.6 \%$ of participants responded correctly. When the experimenter deliberately omitted or repeated a few numbers during counting, question 8 asked participants to tell whether this was correct counting or not. The result suggested $46.7 \%$ of them could tell it was wrong and accurately pointed out where the mistake happened, while $10.3 \%$ of them knew it was wrong but did not know the reason, and $43.0 \%$ could not tell at all. From the test results of question 5 and 7, it was shown that rural children in their early school years have established a preliminary cardinality concept that could help them count with the assistance of real objects. On the other hand, the result of question 6 and 8 proved that children at this stage did not really have a good understanding of the "cardinality concept".

\subsubsection{Ordinal Number Concept}

The full marks of this section were 5 points, and the test results showed an average score of 3.23 and standard deviation 1.42. Similar to the cardinality concept test results, this test proved that rural children at this age understood the ordinal number up to a point. For instance, in question 9, where participants were asked to point out the position of a certain button in a row (eg. the 5 th), $68.2 \%$ of them responded correctly; but in question 10 where they were asked to put a button in a certain number's place, only $29.9 \%$ of them did it accurately. $56.1 \%$ misunderstood the "ordinal number" in this question, because when asked to "put the button in the sixth place", some participants put the button behind the sixth which made it become the seventh, or some replaced it with the original sixth one. 


\subsubsection{Quantitative Comparison}

The quantitative comparison section, which aimed to test children's ability to compare the quantity of objects and numbers, had full marks of 9 points in 3 questions, an average score of 3.47, and a standard deviation of 2.88. Before testing participants, the experimenter would first show them a scale of numbers, 1-10, 10-20, and 20-30 respectively. Then the examiner would present two identical cards with numbers, and ask participants which number in the card was "closer" to a certain number. For example, the examiner showed the participant a scale of 1-10 and 2 cards, one with the number 4 , one with 9. Then the participant was asked "between 4 and 9, which number is closer to number 7?" In each question, the examiner showed 3 different sets of numbers for participants to compare. In the questions, $14.0 \%$ of participants got all 3 comparisons correct, while 38.3\% got them all wrong. In the questions about the scale 10-20, the percentage of participants who answered all correctly was $37.4 \%$, all wrongly $38.3 \%$. In the question about the scale of $20-30$, only $13.1 \%$ of participants got the correct answer, but $40.2 \%$ of them did not, and another $34.3 \%$ said they could not understand what they were being asked to do. The result suggested that children at this stage had difficulty understanding the concept of being "closer" or "further". A further study of the participants showed that this phenomenon was related to their mandarin ability. In addition, the results of this test presented a quite severely polarized situation of these children's quantity comparison abilities.

\subsubsection{Quantity Conservation Concept}

This section with 3 questions about quantity conservation concept had full marks of 3 points, and the results showed an average score of 1.08 and standard deviation of 0.87 . In question 12, participants were shown two sets of buttons (see Figure 2 below), and were asked which set had more buttons. 22.4\% of them answered correctly, which meant they had a preliminary understanding of the quantity conservation concept. In question 13, participants were asked which pattern had more buttons (see Figure 3 below), and $45.8 \%$ of them could see that all four had the same number in spite of being arranged in different shapes and density. Question 14 only allowed participants to count the white buttons aligned with the black ones (see Figure 4 below), and then were asked the number of black buttons after 2 of them were taken away. In this question, $40.2 \%$ of them got the right number. Compared with the result of question 12, it could be surmised that children performed better in conventional graphics than in abstract ones due to their limited abstract thinking and divergent thinking ability.

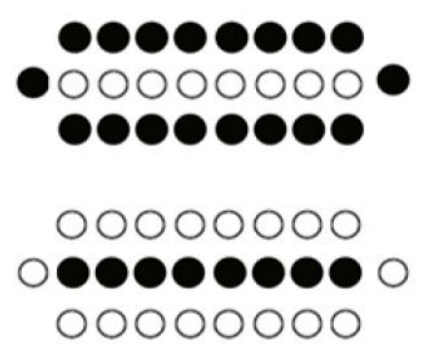

Figure 2. Buttons arrangement in question 12
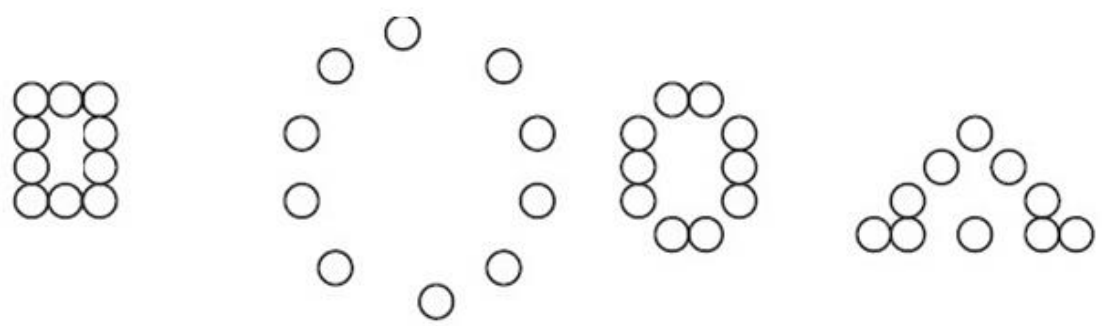

Figure 3. Buttons arrangement in question 13 


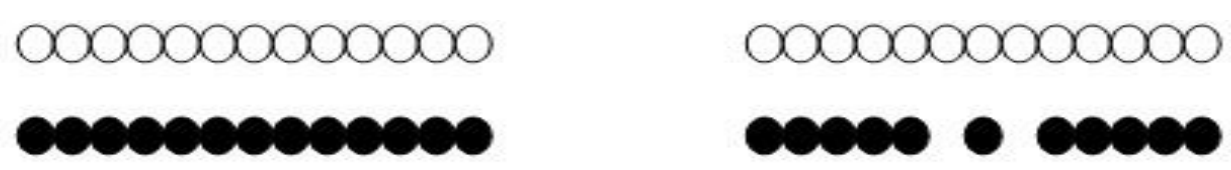

Figure 4. Buttons arrangement in question 14

\subsubsection{Number Operation}

With full marks of 26 points, this section concerning number operation presented an average score of 10.05 and standard deviation of 5.62. In questions about addition, participants worked best with one-digit operations, with $83.2 \%$ of them answering correctly. Concerning addition within the number of 20 , the accuracy rate dropped to $24.3 \%$. When the number increased to within 30, the accuracy rated dropped again to $9.3 \%$. In questions about subtraction, $80.4 \%$ of participants managed one-digit operation, while $16.8 \%$ of them answered correctly to within-20 operations and only $4.7 \%$ to within-30 ones. In one-digit multiplication questions, $50.5 \%$ of participants could answer correctly by mental arithmetic since multiplication could be seen as addition too. $14.0 \%$ of them got answers correct by counting with figures. In division questions, only $23.4 \%$ of participants calculated correctly, with $3.7 \%$ counting with figures. This is because children in early school years could have established concepts of equally divided numbers, so they could do simple divisions by using figures. The results in this section suggested that school teaching played an important role in developing children's operation abilities, because most children utilized mental arithmetic to answer questions. Some children would chant out the operation process to help them calculate. A smaller group of children relied too much on physical objects such as fingers, which would slow the development of their abstract thinking ability.

It was shown by the test that rural children in their early school years did not perform well in counting skills, quantitative comparison, the quantity conservation concept, and number operation, which suggested that they have not yet developed abstract thinking, but rather thought in images. According to Jean Piaget's (1936) theory of cognitive development, children at this age are supposed to be at the preoperational and concrete operational stage, and have formed the quantity conservation concept to a certain degree, but the studied participants in this test had not yet reached this level, which demonstrated a delay in development.

\subsection{Results Analysis of the Number-concept Assessment Test for Rural Children in Their Early Primary School Years}

In this study, SPSS 22.0 was used as a statistical tool to sort and analyze the data. The averages and standard deviations were used for description, and box plots were used for comparison. Independent-sample T Test and One-way ANOVA were utilized in analyzing influencing factors such as age, gender, school teaching and language use of rural children in their early primary school years. Tukey's test in Post Hoc Multiple Comparisons was used to analyze the significant differences between three or more variables. The analysis suggested that age and gender lead to no significant differences in number-concept development of children in rural areas, while school teaching and language use showed significant differences in different aspects.

\subsubsection{The Impact of Gender on Children's Number-concept Ability}

Among the participants, there are 90 males and 124 females, taking up $42 \%$ and $58 \%$ of the total number respectively. As shown in Figure 5, the average score for males was 27.97 with a standard deviation of 1.21, while females had a higher average score of 32.16 and standard deviation of 1.23. Since there was a gap between the two groups, the independent sample $\mathrm{T}$ test was used to examine the difference between the gender and the number-concept of early school-age children in rural areas. The results showed that homogeneity of variance of gender was not significant to children's number-concept ability ( $\mathrm{p}>0.05)$. Among the 6 sections, namely number counting skills ( $\mathrm{T}=-2.23$, $\mathrm{df}=212, \mathrm{p}<0.05)$, cardinality concept $(\mathrm{T}=-1.75, \mathrm{df}=212, \mathrm{p}>0.05)$, ordinal number concept $(\mathrm{T}=-0.49, \mathrm{df}=212, \mathrm{p}>0.05)$, quantitative comparison $(\mathrm{T}=0.29, \mathrm{df}=212, \mathrm{p}>0.05)$, quantity conservation concept $(\mathrm{T}=-2.18, \mathrm{df}=212, \mathrm{p}<0.05)$, and number operation $(\mathrm{T}=0.27, \mathrm{df}=212, \mathrm{p}<0.05)$, and the total score $(\mathrm{T}=-2.36, \mathrm{df}=212, \mathrm{p}<0.05)$, it is clear that different genders performed significantly different in counting skills, quantity conservation concept, number operation, and the total score, while no statistical differences were found in the other 3 sections. These findings also demonstrated girls performed slightly better than boys but with larger individual differences. One possible explanation is that girls' language development is generally faster than boys, which also illustrates the role that languages might play in cognitive development. Another possible reason is that boys tend to be more lively than girls and feel that schools restrict their activities, so they need a longer period to adapt. 


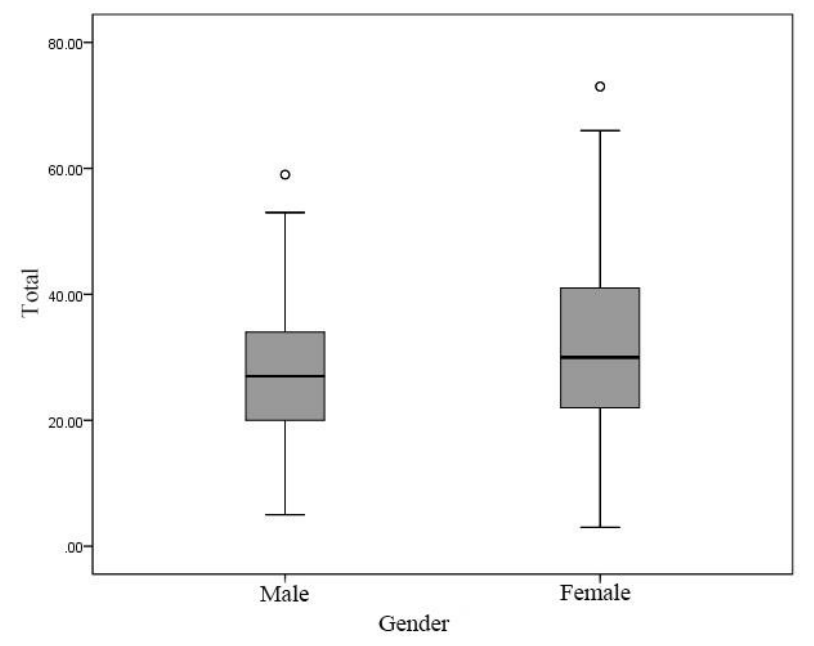

Figure 5. Comparison of participants' total score for different genders

\subsubsection{The Impact of Age on Children's Number-concept Ability}

In the test, there were 80 children at the age of 6,116 at age 7 , and 18 at age 8 . As shown in Figure 6 , the youngest group got an average score of 27.58, with standard deviation of 1.22; the average score of children at 7 was 32.45 and their standard deviation was 1.27 , while the same indicators for children at 8 were 29.78 and 3.34 . These statistics show that 7-year-old children performed slightly better than the others and 8-year-olds had large individual differences. Through One-Way ANOVA, it could be seen that age is the main factor in children's number-concept development $(\mathrm{F}=3.47, \mathrm{p}<0.05)$. Table 1 also displays significant differences between different ages in the score of counting skills and number operation, but not in the rest of the items. LSD multiple post hoc comparisons were used to examine the differences between the three ages. In the total score, there were significant differences between 6 and 7 year olds $\left(\mathrm{MD}_{(6-7)}=-4.87, \mathrm{p}<0.01\right)$, but the total score of 6 and 8 year olds $\left(\mathrm{MD}_{(6-8)}=-2.20, \mathrm{p}>0.05\right)$, and 7 and 8 years old $\left(\mathrm{MD}_{(7-8)}=2.67, \mathrm{p}>0.05\right)$ were not significantly different. This means that 7 years old is the critical period for the development of the number concept. Children who miss this critical period might lag behind when they enter primary school (Note 2). It can also be argued that the age of 7 is equally important for psychological development. If school teaching is not adequate during this critical period, children's educational outcomes may be diminished.

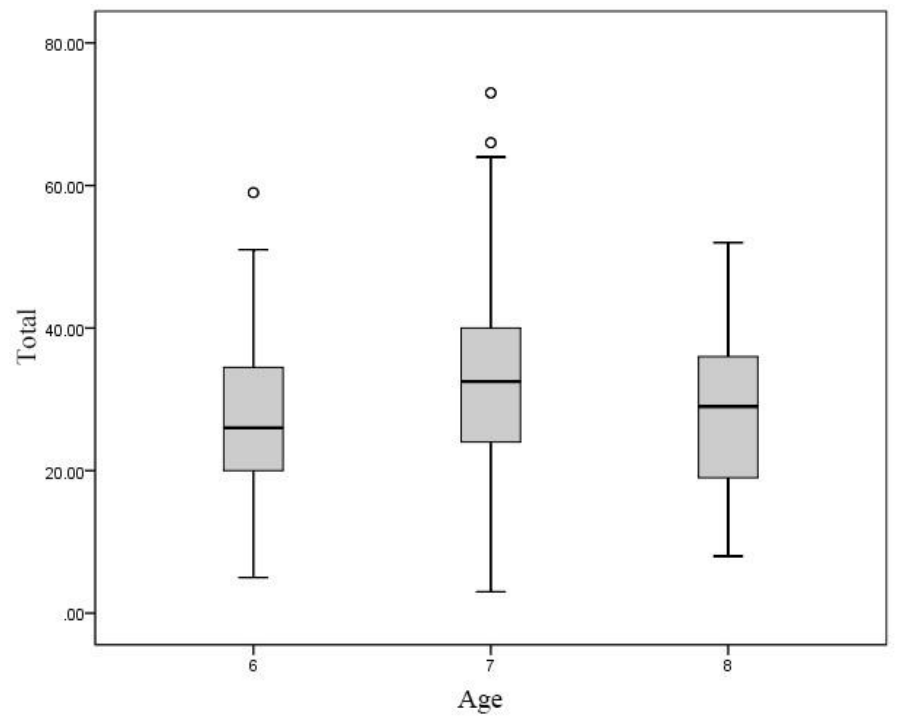

Figure 6. Comparison of participants' total score for different ages 
Table 1. Analysis of the different variables in influencing participants' mathematical ability

\begin{tabular}{|c|c|c|c|c|c|c|c|c|}
\hline \multirow[b]{2}{*}{ item } & \multicolumn{2}{|c|}{ Age } & \multicolumn{2}{|c|}{ School } & \multicolumn{2}{|c|}{ Language foundation } & \multicolumn{2}{|c|}{ Language usage } \\
\hline & $\mathrm{F}$ & $\begin{array}{c}\text { Significant } \\
\text { level }\end{array}$ & $\mathrm{F}$ & $\begin{array}{c}\text { Significant } \\
\text { level }\end{array}$ & $\mathrm{F}$ & $\begin{array}{c}\text { Significant } \\
\text { level }\end{array}$ & $\mathrm{F}$ & $\begin{array}{c}\text { Significant } \\
\text { level }\end{array}$ \\
\hline Total score & 3.47 & $0.03 *$ & 1.92 & 0.15 & 1.63 & 0.20 & 11.22 & $0.00 * * *$ \\
\hline Counting skills & 3.91 & $0.02 *$ & 2.09 & 0.13 & 3.00 & 0.05 & 9.64 & $0.00 * * *$ \\
\hline Cardinal concept & 0.05 & 0.96 & 18.54 & $0.00 * * *$ & 3.33 & $0.04 *$ & 19.71 & $0.00 * * *$ \\
\hline Ordinal concept & 0.28 & 0.76 & 1.59 & 0.21 & 1.33 & 0.27 & 16.33 & $0.00 * * *$ \\
\hline Quantitative comparison & 1.87 & 0.16 & 5.64 & $0.004 * *$ & 2.06 & 0.13 & 1.48 & 0.23 \\
\hline $\begin{array}{l}\text { Quantity conservation } \\
\text { concept }\end{array}$ & 1.21 & 0.30 & 11.6 & $0.00^{* * *}$ & 3.90 & $0.02 *$ & 0.93 & 0.40 \\
\hline Number operation & 4.44 & $0.03 *$ & 1.52 & 0.22 & 1.95 & 0.15 & 7.39 & $0.001 * *$ \\
\hline
\end{tabular}

$* \mathrm{P}<0.05 ; * * \mathrm{P}<0.01 ; * * * \mathrm{P}<0.001$

\subsubsection{The Impact of School on Children's Number-concept Ability}

Participants in this test were from three rural schools in Y province, 34 from village school B, 138 from town school C, and 42 from village school D. Among the three, C and D were both town level schools, so they were equipped with better teaching resources and have a 6-grade system while B was a village school which only had a 3-grade system. When children progressed to grade 4, they would be transferred to a town school. Figure 7 shows that the average score for school B was 27.00 and its standard deviation 2.31; the same figures for school C were 29.29 and 2.39, school D 31.59 and 1.01. Therefore, it is clear that school B scored the lowest. Through One-Way ANOVA, Table 1 clearly presented the significant differences between the 3 schools, especially in the cardinal concept $(\mathrm{F}(2,211)=18.54, \mathrm{p}<0.001)$, quantitative comparison $\left(\mathrm{F}_{(2,211)}=5.64, \mathrm{p}<0.01\right)$, and quantity conservation concept $\left(\mathrm{F}_{(2,211)}=11.76, \mathrm{p}<0.001\right)$. There were no significant differences in the other factors. It is preliminarily demonstrated that school has a profound impact on children's number concept development.

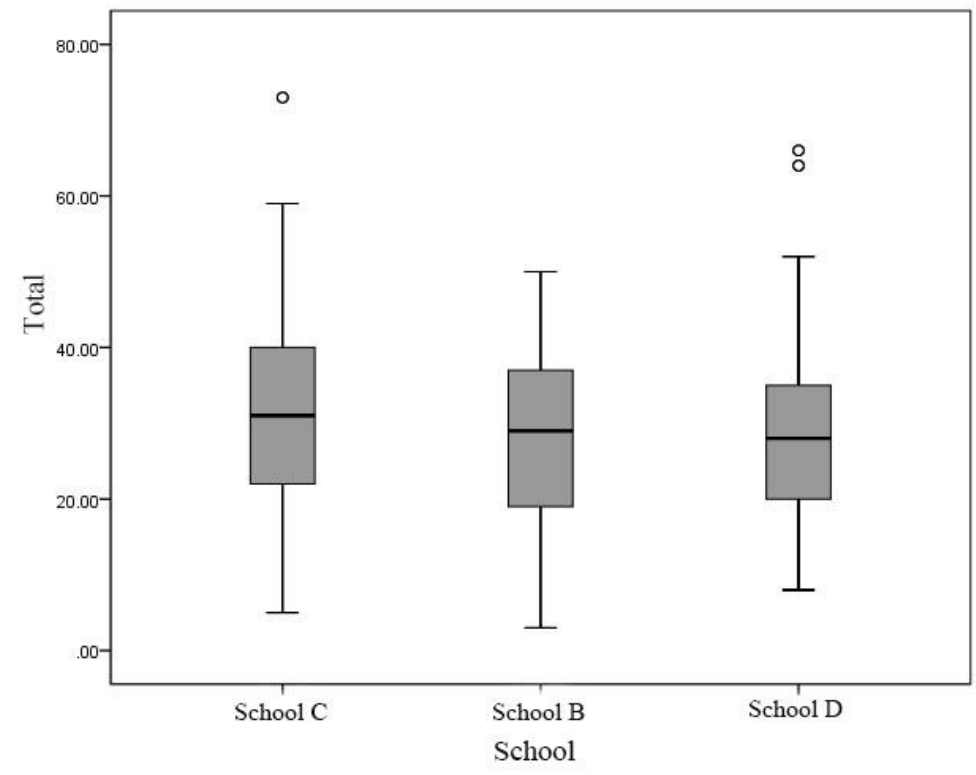

Figure 7. Comparison of participants' total score for different schools

\subsubsection{The Impact of Language on Children's Number-concept Ability}

In this study, the language ability at enrollment had been divided into three types: $50.47 \%$ of participants only speaking a minority language, $44.86 \%$ only speaking Mandarin, and $4.67 \%$ speaking both. As is shown in Figure 8 , the average score of children speaking only a minority language was 30.68 and the standard deviation 1.42, while the results for mandarin-only speaking were 29.40 and 1.12 , for bilingual 37.00 and 1.92 . Therefore, it is clear that bilingual children performed better in the number-concept assessment test than the other two groups. This result supports the previous literature. Further checks by One-Way ANOVA in Table 1showed that there was no significant relevance between children's language use before entering school and their number-concept development, with the exception of performance in cardinal concept $(\mathrm{F}=3.33, \mathrm{p}<0.05)$ and quantity conservation $(\mathrm{F}=3.90, \mathrm{p}<0.05)$. Interviews with the families of participants who scored higher found they could already use numbers in their everyday conversations before 
they entered primary school. They could form complete sentences with numbers in daily life contexts (for example, "I can see three birds in the sky, and 2 of them are black".), or in calculating contexts (for example, "how many birds are there? One, two, three".), which could potentially help number-concept development. On the other hand, interviews with the families of participants who scored quite low showed that they lack language stimulation before they entered primary school.

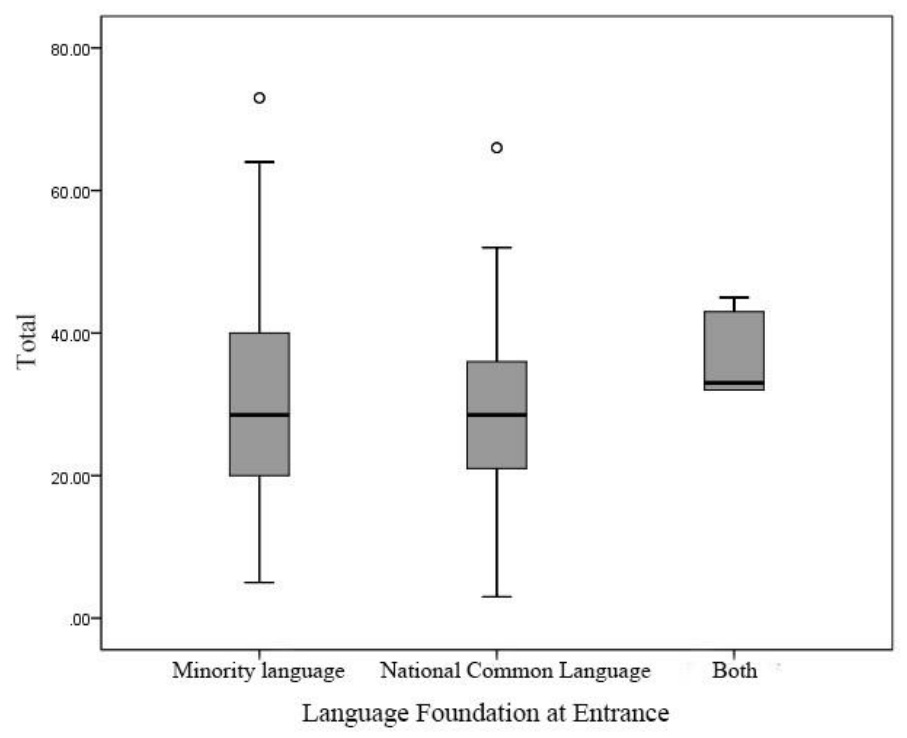

Figure 8. Comparison of participants' total score for different language abilities at entrance of school

Language ability after the entrance to primary school is also an important factor for number-concept development. Data had also been collected about the language that participants used to communicate with teachers and classmates after enrollment. $15.2 \%$ of them used their ethnic languages in schools, while $81.9 \%$ spoke mandarin in schools, and $2.9 \%$ mainly spoke ethnic language in school with Mandarin as a supplement. Figure 9 shows that the first group of participants scored an average of 20.94 with a standard deviation of 2.06; the average score of children speaking Mandarin was 31.76 with a standard deviation of 0.93 ; for children who used both languages in school but mainly an ethnic language, the two figures were 26.67 and 2.38. It can be seen that the second group, who used Mandarin after entering school, performed significantly better than the other two groups who used their own ethic languages as the main language. The fact that schools use Mandarin in teaching may account for this. Ethnic-language-speaking children's need for language shift affected their understanding of mathematical knowledge, and thus delayed the development of the number concept to some extent. To further check the impact of language used after entering primary school on children's number-concept development, One-Way ANOVA was used as shown in Table 1. Significant differences appeared in every section including the total score with the only exceptions being quantitative comparison and the quantity conservation concept. LSD multiple post hoc comparisons were used to check the difference among the three language variables as well. It was found that the main difference in the overall score was reflected in the ethnic language and Mandarin $\left(\mathrm{MD}_{\text {(ethnic languge-mandarin) }}=-10.83, \mathrm{p}<0.01\right)$. Therefore, encouraging the use of mandarin in pre-school can promote the development of children's number-concept. 


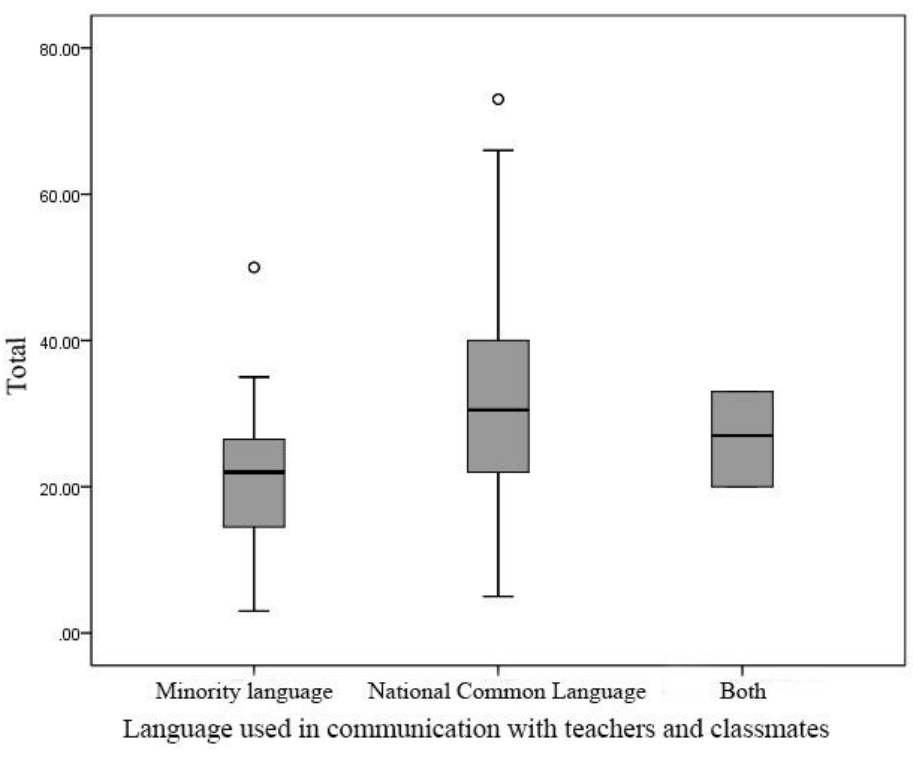

Figure 9. Comparison of participants' total score for different languages used after enrollment

\section{Discussion on Features of Number-concept Development of Rural Children in Early Primary School Years}

\subsection{Overall Development Lagging Behind}

Children in their early primary school years have already learned to use symbols to represent numbers 1, 2, 3, 4, 5 and so forth, and also understood the meaning of "one person", "two persons". They then made a major inductive leap and gradually understood that the last word in the count represented the cardinality of the entire set. This concept is called the cardinal principle (Gelman \& Gallistel, 1978). To fully grasp this concept, children must first understand all aspects of the integer concept expressed by cardinal numbers. For instance, children have to find the correlation between numbers and quantification (Bloom \& Wynn, 1997; Gelman \& Gallistel, 1978), and they have to understand numerosity to extend number words from one set to another (Slusser \& Sarnecka, 2011).

The early primary school years are a key period for learning how to use numbers to understand mathematical relationships, that is, to acquire number counting skills, and knowledge of the cardinality concept, ordinal number concept, quantitative comparison, quantity conservation concept, and number operation. Children in rural areas lag behind in these areas because their language ability has not been fully developed in the critical period of development, and therefore they cannot process preliminary abstract symbols. Senzaki's research suggested language-related factors interact with age-specific factors (Senzaki, Lanter, \& Shimizu, 2019). Piaget's theory pointed out that the early school age (about 6 and a half years old) is a critical period for children to form the concept of quantity conservation, and the development process of children's thinking is gradually transitioning from image thinking to abstract thinking (Piaget, 1997). Moreover, Zhu Zhixian proposed that children in the lower grades (7-8 years old) are able to form a preliminary concept system of integers within three digits (Zhu, 1989). However, although the average age of the participants in this study was 6.71 , most of them had not even mastered integers to 100, which proved that rural children's number-concept development was lagging behind in their early school years. In addition, in the process of the test, more than $80 \%$ of children completed the test with the help of their fingers, the over-reliance on which indicated that rural children in early primary school were still very reliant on visual thinking. Most children had not developed the quantity conservation concept, so the overall number concept was lagging.

\subsection{School Teaching Quality Affecting Development}

Among the three schools, C and D had advantages in teaching methods, teaching conditions, and teachers' qualifications over B. As a result, participants in school B scored lower than those in the other two, especially in cardinality concept and quantity conservation concept, which indicates that school teaching affected children's number-concept development. In the process of the test, some students complained, "I haven't learned about this, so I don't know how to do it". In fact, pre-school education in this area mainly functioned as childcare, while the educational function had been put on hold due to factors such as insufficient teachers, limited funding, and parents' lack of attention. This has seriously affected the development of children's number-concept and other capabilities in the key preschool period, which further validates the early hypothesis in this test that the lack of preschool education does 
hinder the development of children's number-concept development. After entering primary school for formal and systematic learning, study has become a child's leading activity and has promoted children's psychological development. Therefore, rural children's number- concept development in early school years is significantly affected by the quality of school teaching. The differences in the score between ages in this test were not significant, and all participants' scores were comparatively low. It can be concluded that the individual's own development did not help the formation of children's number-concept as much as school teaching did.

\subsection{Family Factors Playing a Role}

Another hypothesis in this test was that a multi-language environment can facilitate the development of children's number-concept, but the results show that for rural children in their early school years, a multi-language environment alone is not sufficient. Mathematical vocabulary used before entering school is pivotal. Kindergarten and carers of children in the family should both be held responsible in this respect. In rural areas, most children's parents go out to work, so those children were left-behind and taken care of by the elderly, such as grandparents who might be illiterate or less educated. They are often not aware of the importance of language stimulation or preschool education. In those areas, children's lack of parental companionship and education, together with other social, demographic, and economic factors, lead to the lagging development of children's education. Therefore, although the development of number-concept is affected by school and family factors, macro factors that affect the quality of school teaching and family environment should also be considered, and may provide impetus for future research.

\section{Strategies for Number-Concept Development of Rural Children in Early School Years}

\subsection{Facilitating Language Development to Promote the Number-concept}

The first few years of school are a crucial period for developing the concept of numbers. In recent years, the importance of developing theoretical and empirical foundations to test the relationship between language, culture and thought has been emphasized (Imai, Kanero, \& Masuda, 2016; Imai \& Masuda, 2013), because different language environments can lead to different development processes (Senzaki et al., 2019). Several theories suggested that the development of the number-concept is supported by language syntax. Carey (2009) thought "long-term memory models that support the meanings of singular, dual, and triple markers, as well as the child's first numbers... must be created in the course of language learning". Barner and his colleagues proposed another syntactic-semantic guidance theory, and they found that children's comprehension of quantifiers were related to how well caregivers understand and explain them as well as vocabulary level, regardless of age. They held that quantifiers may facilitate numeral acquisition by providing grounding in the semantic function of numerals, and therefore, "learning quantifiers may make the general hypothesis space of sets and individuals more salient as a hypothesis space for integer acquisition" (Barner, Chow, \& Yang, 2009). Numerical concept acquisition has also been described with another language-based guide. Conceptual role guidance gradually builds the full meaning of a symbol by allowing children to manipulate symbols to handle only partially expressed concepts (Carey, 2009). Understanding vocabulary may help children acquire numerical knowledge by eliminating noun phrases that follow some numeric words. When numbers are used to quantify things that exist in a child's environment, the knowledge of the noun already provides a partial explanation for the numeric phrases. For example, when a child hears "look! That's mother duck with three ducklings!" If they have already understood the noun "duck", it is easier for them to comprehend the number "three" since knowledge of nouns supports number learning in this way. This correlation should be taken advantage of to promote the development of children's number-concept.

\subsection{Combining the Characteristics of Number-concept Development to Stimulate Children's Learning Interest}

Interest in learning has a significant impact on students' academic achievement, especially for children who are in the early school years when they are transitioning from visual thinking to abstract thinking. The rural children in this study still relied on visual thinking, lagging behind their peers. Therefore, in teaching, it is advisable to consider children's thought processes, choosing teaching methods and tools that are suitable for their cultural background and life experience, designing games close to their daily life, and letting students feel a sense of intimacy and familiarity in school teaching so as to promote students' number-concepts. As long as they can experience a sense of accomplishment in learning, children will maximize their interest in mathematical learning, and then lay a better foundation to grasp knowledge. In addition, teachers can develop and create culturally rich courses suitable for rural children, such as courses concerning their hometown and school under the overall planning of the government. It will also be beneficial if they can design math textbooks specially written for rural areas, which more closely align to rural children's lives, in order to pass on culture, increase their interest in learning, and boost their self-confidence. The combination of curriculum content and traditional culture will increase children's motivation to learn math.

\subsection{Attaching More Importance to Pre-school Education in Rural Areas}

The public kindergartens in the area where the test was conducted are concentrated in towns, while village 
kindergartens are co-organized with primary schools. Due to insufficient teachers and poor facility conditions, this kind of "kindergarten" is closer to a nursery school and its educational function is effectively lost. Many children were sent to these "kindergartens" in the village after turning 1 year old, while their parents went to the major cities for work. Further, in these rural areas, children have insufficient teaching resources, and the teaching quality is relatively low; the lack of education in "kindergartens" makes it difficult for children to develop their thinking after entering the school, thus restraining the development of number concept of local children. Therefore, in view of the current situation, the government and education departments should increase the number of kindergartens and support for preschool education in remote areas or areas with poor teaching quality. The teaching quality in current kindergartens should be improved, and children allocated to the nearest appropriate school. This can enable children in the area to receive effective pre-school education. In addition, more effort should be made to train preschool teachers, so they can help children to build better foundation before the entrance to primary school. Ways to further improve pre-school education in rural areas also needs further research.

\section{Conclusion}

The Number-concept plays a very important role in children's growth, and it is of great significance to the development of children's thinking and mathematical ability. For rural children in early school years, a multilingual environment is an advantage in promoting their number-concept development. However, as families and preschools have not given sufficient guidance, these children's number-concept development still lags behind in their early school years. This study found the following solutions to be effective: first, teachers and families should work together on enlarging children's vocabulary; second, both parties need to cultivate children's own interest in mathematics; third, government policy support is necessary to improve the preschool education mechanism. The connection between number-concept development and language development was also identified for more detailed investigation in the future.

\section{Acknowledgement}

This work was supported by the Educational Youth Project 2019 in the "13th Five-Year Plan" of the National Social Science Fund of China (grant number: CMA190246).

\section{References}

Ansari, D., Donlan, C., Thomas, M. S. C., Ewing, S. A., Peen, T., \& Karmiloff-Smith, A. (2003). What makes counting count? Verbal and visuo-spatial contributions to typical and atypical number development. Journal of Experimental Child Psychology, 85(1), 50-62. https://doi.org/10.1016/s0022-0965(03)00026-2

Barner, D., Chow, K., \& Yang, S. J. (2009). Finding one's meaning: A test of the relation between quantifiers and integers in language development. Cognitive Psychology, 58(2), 195-219. https://doi.org/10.1016/j.cogpsych.2008.07.001

Baroody, A. J., \& Ginsburg, H. P. (1982). Preschoolers' Informal Mathematical Skills: Research and Diagnosis. American Journal of Diseases of Children, 136(3), 195-197. https://doi.org/10.1001/archpedi.1982.03970390009001

Carey, S. (2009). The Origin of Concepts. New York: Oxford University Press. https://doi.org/10.1093/acprof:oso/9780195367638.001.0001

Duncan, G. J., Dowsett, C. J., Claessens, A., Magnuson, K., Huston, A. C., Klebanov, P., ... Japel, C. (2007). School readiness and later achievement. Developmental Psychology, 43(6), 1428-1446. https://doi.org/10.1037/0012-1649.43.6.1428

Gelman, R., \& Gallistel, C. R. (1978). The child's understanding of number. Cambridge MA: Harvard University Press.

Ginsburg, H. P., \& Baroody, A. J. (1990). Test of Early Mathematics Ability (2nd ed.). Pro-Ed (Firm). Retrieved from https://www.researchconnections.org/childcare/resources/3220

Greenes, C., Ginsburg, H. P., \& Balfanz, R. (2004). Big Math for Little Kids. Early Childhood Research Quarterly, 19(1), 159-166. https://doi.org/10.1016/j.ecresq.2004.01.010

Griffin, S. A., Case, R., \& Siegler, R. S. (1994). Right start: Providing the central conceptual prerequisites for first formal learning of arithmetic to students at risk for school failure. In Kate McGilly (Ed.), Classroom lessons: Integrating cognitive theory and classroom practice (pp. 25-49). Cambridge MA \& London, England: The MIT Press.

Howden, H. (1989). Teaching Number Sense. The Arithmetic Teacher, 36(6), 6-11. Retrieved from www.jstor.org/stable/41194455

Imai, M., \& Masuda, T. (2013). The Role of Language and Culture in Universality and Diversity of Human Concepts. 
In M. Gelfand, C.-Y. Chiu, \& Y.-Y. Hong (Eds.), Advances in Culture and Psychology (Vol. 3). New York: Oxford University Press. https://doi.org/10.1093/acprof:oso/9780199930449.003.0001

Imai, M., Kanero, J., \& Masuda, T. (2016). The relation between language, culture, and thought. Current Opinion in Psychology, 8, 70-77. https://doi.org/10.1016/j.copsyc.2015.10.011

Malofeeva, E., Day, J., Saco, X., Young, L., \& Ciancio, D. (2004). Construction and Evaluation of a Number Sense Test With Head Start Children. Journal of Educational Psychology, 96(4), 648-659. https://doi.org/10.1037/0022-0663.96.4.648

Piaget, J. (1936). Origins of intelligence in the child. London: Routledge \& Kegan Paul.

Piaget, J. (1997). The Language and Thought of the Child (M. Gabain \& R. Gabain, Trans.). London: Routledge.

Senzaki, S., Lanter, J., \& Shimizu, Y. (2019). The development of attention to singular vs. plural sets in preschool children: Insights from a cross-linguistic comparison between English and Japanese. Cognitive Development, 52, 100810. https://doi.org/10.1016/j.cogdev.2019.100810

Slusser, E. B., \& Sarnecka, B. W. (2011). Find the picture of eight turtles: A link between children's counting and their knowledge of number-word semantics. Journal of Experimental Child Psychology, 110(1), 38-51. https://doi.org/10.1016/j.jecp.2011.03.006

Starkey, P., \& Klein, A. (2000). Fostering Parental Support for Children's Mathematical Development: An Intervention with Head Start Families. Early Education and Development, 11(5), 659-680. https://doi.org/10.1207/s15566935eed1105_7

Xie, Y. K., Tian, X. J., \& Lv, X. (2014). The Bottleneck and Breakthrough of Preschool Education Development in Southwestern Rural Ethnic Areas. Studies in Early Childhood Education, 04, 58-63. https://doi.org/10.13861/j.cnki.sece.2014.04.009

Zhao, M, \& Jiang, F. (2011). The Predicament and Solution of Preschool Education in Rural Ethnic Areas. Forum on Contemporary Education, 05, 50-52.

Zhao, Z. G., \& Zhou, X. (2006). Research on Development of Estimation and Number-concept of Children Aged 3-6 (Master's thesis, East China Normal University, Shanghai, PR China). Retrieved from China National Knowledge Infrastructure.

Zhu, Z. X. (Ed.). (1989). Encyclopedia of Psychology. Beijing: Beijing Normal University Press.

\section{Notes}

Note 1. In China, cities do not only include urban areas; They also include towns and villages which surround the city proper. These are considered to be rural areas.

Note 2. In China, primary school generally begins at the age of 6 , but this can vary in rural areas due to less strict requirements. The average age of primary school entrants in this study was 7 .

\section{Copyrights}

Copyright for this article is retained by the author(s), with first publication rights granted to the journal.

This is an open-access article distributed under the terms and conditions of the Creative Commons Attribution license which permits unrestricted use, distribution, and reproduction in any medium, provided the original work is properly cited. 